\title{
Symmetrical polyarthritis is not always rheumatoid
}

\author{
N Menon, R Madhok
}

\section{Case report}

A 63 year old woman was referred for further management of seronegative rheumatoid arthritis. She had been well until 16 weeks before admission when she developed abrupt onset pain, swelling and stiffness of her hands, feet, ankles and knees. In addition she complained of lethargy, malaise and weight loss which she could not quantify. She was admitted to her local hospital where the presence of synovitis was noted, no other abnormalities were noted on general examination on four repeated occasions by two general physicians. A diagnosis of rheumatoid arthritis was made (rheumatoid factor: negative; antinuclear factor: negative) and treatment with nonsteroidal anti-inflammatory agents and analgesics begun. She failed to improve despite optimal therapy and was referred for second line treatment.

She had no previous illnesses requiring hospital referral. There were no associated symptoms or signs suggestive of mucocutaneous, eye or bowel disease.

Examination revealed a thin, pale individual with evidence of weight loss. Generalised lymphadenopathy was noted in the submental, cervical, both axillary, left epitrochlear and both inguinal regions. She had marked synovitis of her knees, ankles and the metacarpophalangeal and interphalangeal joints of both hands. The liver was enlarged $3 \mathrm{cms}$ and the spleen $2 \mathrm{cms}$ below the costal margin. She had an ejection systolic murmur over the precordium with radiation to the carotid areas. The chest was clear and the central nervous system was clinically normal.

Initial investigations revealed a haemoglobin of $9.3 \mathrm{~g} / \mathrm{dl}$, white cell count of $4.9 \times 10^{9} / 1$ and platelet count of $314 \times 10^{9} / 1$. Blood urea was $3.9 \mathrm{mmol} / \mathrm{l}$, serum creatinine $67 \mu \mathrm{mol} / \mathrm{l}$, total protein $57 \mathrm{~g} / \mathrm{l}$, albumin $35 \mathrm{~g} / \mathrm{l}$, alk phosphatase $242 \mathrm{u} / \mathrm{l}, \operatorname{IgG} 8.7 \mathrm{~g} / 1, \operatorname{IgA} 1.0 \mathrm{~g} / 1$ and $\operatorname{IgM}$ $2.5 \mathrm{~g} /$. The ESR was $7 \mathrm{~mm}$ in the first hour and the CRP was $<10 \mathrm{mg} / \mathrm{l}$. The rheumatoid factor and antinuclear factor were again negative. Blood and urine cultures were negative. Serology for cytomegalovirus, Epstein-Barr virus and toxoplasma was negative. Radiographs showed possible early erosive changes at the metacarpophalangeal and metatarsophalangeal joints. A chest radiograph showed small bilateral pleural effusions; no cardiomegaly or mediastinal lymphadenopathy was noted. A CT scan of the abdomen and pelvis confirmed hepatomegaly with no focal lesion, and enlarged lymph nodes were noted.

Biopsy of a left axillary lymph node showed effacement of architecture with predominence of large pleomorphic lymphoid cells with clear cytoplasm, smaller lymphoid cells and histiocytic cells giving a granulomatous appearance. Immunocytochemistry showed positive staining of tumour cells with $T$ cell markers in keeping with a high grade $\mathrm{T}$-cell lymphoma. A bone marrow aspirate and trephine showed lymphomatous involvement of the marrow.

Treatment with appropriate cyclical chemotherapy was then started.

\section{Discussion}

Polyarthritis in the elderly must be viewed with suspicion. Although rheumatoid arthritis may frequently present acutely with systemic features for the first time in the elderly, the presence of lymphadenopathy and hepatosplenomegaly should also raise the possibility of an underlying lymphoproliferative disorder. Localised or generalised lymphadenopathy is known to occur in most autoimmune disorders and has been reported in about $50 \%$ of patients with rheumatoid arthritis as part of a 'reactive' phenomenon. ${ }^{1}$ The epitrochlear, axillary, femoral and inguinal areas are commonly involved; in contrast to SLE, cervical involvement is uncommon. Interestingly, about $20 \%$ of patients with generalised reactive lymphadenopathy have been found to develop an inflamatory polyarthritis within one year of biopsy. ${ }^{2}$ Lymphoma, developing in a patient with longstanding rheumatoid arthritis may also present with generalised lymphadenopathy and hepatosplenomegaly but in such patients the arthritis is often of long duration. ${ }^{3}$ Diagnostic dilemma occurs when a lymphoproliferative disorder presents as a polyarthritis. Our patient was initially thought to have rheumatoid arthritis and was treated as such. However, the presence of hepatosplenomegaly associated with generalised lymphadenoathy was suspicious and a lymph node biopsy and bone marrow examination revealed the presence of a lymphoma. A synovial biopsy to determine the nature of the synovitis was unfortunately not undertaken.

Involvement of the musculoskeletal system occurs in $7-25 \%$ of patients with nonHodgkin's lymphoma. Although the commonest problem is bone pain from metastasis or 
lymphoma arising from bone, lymphomas infiltrating the synovial membrane can simulate classical rheumatoid arthritis. ${ }^{4}$ Lymphadenopathy and hepatosplenomegaly may not always be initially apparent and diagnostic suspicion should be aroused by mono- or polyarthritis in patients with disproportionately severe constitutional symptoms or anaemia, particularly if tests for rheumatoid factors are negative. ${ }^{5}$ Radiographs may be misleading since involvement of the bone may cause lytic lesions similar to erosions seen in rheumatoid arthritis. Synovial biopsies in such situations may either reveal the underlying neoplasm ${ }^{4}$ or show reactive changes. ${ }^{6}$

The entity of carcinomatous polyarthritis has also been described in association with tumours of the lung, colon and breast. A rheumatoid-like seronegative arthritis usually develops insidiously in temporal relationship to the tumour, improves with treatment of the underlying tumour and recurs when the tumour reappears. ${ }^{4}$

\section{The lesson}

It is important to conduct a thorough clinical examination on all patients presenting with a polyarthritis. In such patients the presence of a disproportionate anaemia, constitutional symptoms or a seronegative arthritis should arouse suspicions of an underlying malignancy particularly in the older patient.

1 Robertson M D, Hart F D, White W F, Nuki G, Boardman P L. Rheumatoid lymphadenopathy. Ann Rheum Dis 1968; 27: 253-60.

2 Kelly C A, Malcom A J, Griffiths I. Lymphadenopathy in rheumatic patients. Ann Rheum Dis 1987; 46: 224-7.

3 Symmons D P M, Ahern M, Bacon P A, et al. Lymphoproliferative malignancy in rheumatoid arthritis: Lymphoproliferative malignancy in rheumatoid arthritis

4 Dorfman H D, Siegel H L, Perry M C, Oxenhandler R. Non-Hodgkin's lymphoma of the synovium simulating rheumatoid arthritis. Arthritis and Rheumatism 1987; 30: 155-61.

5 Caldwell D S. Musculoskeletal syndromes associated with malignancy. In: Kelley $\mathrm{W} \mathrm{N}$, et al, eds. Textbook of rheumatology, 4th ed. Philadelphia: W B Saunders, 1993.

6 Seleznick M J, Aguilar J L, Rayhack J, Fenske N, Espinoza L R. Polyarthritis associated with cutaneous T cell lymphoma. F Rheumatology 1989; 16: 1379-82. 